\title{
A Comprehensive Daily Activity-Travel Generation Model System for Workers
}

\author{
Chandra R. Bhat \\ University of Texas at Austin \\ and \\ Sujit K. Singh \\ University of Massachusetts at Amherst
}

\begin{abstract}
This paper develops a comprehensive representation to describe the activity-travel pattern of workers and proposes an analysis framework to model the activity-travel attributes identified in the representation. The analysis framework is based on a descriptive examination of activity-travelpatterns of workers from two locations in the U.S. The paper also formulates an econometric methodology to estimate the component of the analysis framework involving the joint modeling of evening commute mode choice, number of evening commute stops, and number of stops after arriving home from work. The methodology is applied to an empiricalanalysis using data from an activity survey conducted in the Boston Metropolitan area and the effects of a variety of congestion-alleviation measures are examined.
\end{abstract}




\title{
A Comprehensive Daily Activity-Travel Generation Model System for Workers
}

\author{
Chandra R. Bhat and Sujit Singh
}

\section{Introduction}

The increasing concern in many urban and suburban areas about the level of traffic congestion, combined with the conceptual deficiencies of the conventional trip-based travel demand modeling approach, has led to the emergence of the activity-based approach to studying travel behavior. The activity-based approach views travel as a derived demand; derived from the need to pursue activities distributed over space. The approach focuses on sequences or patterns of activity behavior, with the whole day or longer periods of time as the unit of analysis. It emphasizes the effects of economic, gender, social, transportation network, and locational divisions on spatial and temporal aspects of individual movement.

The activity-based holistic perspective of travel can reliably evaluate urban travel demand management policies since it explicitly models activity patterns and considers these patterns to be the fundamental influence on travel decisions (see Guensler 1993 and Stopher 1993). The ability of activitybased travel models to better evaluate urban policies is of substantialvalue due to the emphasis in the past decade on altering travelpatterns in a pro-active manner through changing travelbehavior. Clarke (1986), Hivert et al., (1987), Bhat (1997), Gärling et al., (1994) and Recker and McNally (1986), among other researchers, have used an activity-travel approach to analyze the impact on travel of changes in the transport system, changes in the socio-economic environment, and changes in urban land form. The conclusion from these studies has been that the impact of policy actions is very complex and involves shifting activities (and associated travel) in time and space, and consolidation of activities in a single tour.

The activity-based travelanalysis approach has seen substantialdevelopment in the past few years. The objective of this paper is to contribute to this literature by a) Developing a comprehensive representation of attributes characterizing a worker's daily activity-travelpattern, b) Proposing an analysis framework for modeling the workday activity-travel pattern of individuals, and c) Formulating and applying an econometric methodology to estimate a component of the analysis framework involving the joint modeling of evening commute mode choice, number of evening commute stops, and number of stops after 
arriving home after work. In proposing the analysis framework, we descriptively examine the daily activity travel patterns of workers using data sets from two locations in the U.S.

The rest of this paper is structured as follows. The next section develops a representation of the workday activity-travelpattern of workers. Section 3 analyzes (descriptively) the activity-travelpattern of workers from two metropolitan areas in the U.S. Section 4 proposes an analysis framework for activitytravel pattern modeling. Section 5 focuses on econometric formulation and empirical analysis. The final section concludes the paper.

\section{Workday Activity-Travel Pattern Representation}

We consider household and individual socio-demographics as exogenous determinants of workday activity-travel pattern behavior. The activity-travel environment is also considered as an exogenous input. The activity-travel environment comprises both the transportation system (i.e, the network configuration of roads and the transit system) and the land-use environment (the location of opportunities for activity participation). Conditional on socio-demographics and the activity-travel environment, individuals make medium-term decisions (in combination with other individuals in their household) regarding their employment (whether to be employed, hours of work, start time at work in the morning and end time of work in the evening, location of work place, etc.), residence (type of residence, location, etc.), and car ownership. We will consider these medium-term decisions as being exogenous to the determination of the daily activity-travelpattern (the medium-term activity-traveldecisions may be modeled separately prior to the modeling of the daily activity-travel pattern, see Bhat and Koppelman, 1993). Finally, we will assume $3 \mathrm{am}$ to be the start of the day and will assume that all individuals are at home during the start of the day.

Our representation of a worker's workday activity-travelpattern rests on the regularity and "fixity" of the work activity, and the fixity of the home location. In concept, our approach is similar to the one proposed by Damm (1980) in its use of the work activity as the "peg" to represent the activity-travel pattern. However, the representation developed here is more extensive and complete than the one by Damm who focuses only on two dimensions of activity participation: activity participation choice and 
activity duration. Our representation also extends Hamed and Mannering's (1993) work to accommodate mode choice and number of stops decisions in the activity-travel pattern and generalizes their post-work activity involvement to include the entire day.

Figure 1 provides an overview of the workday activity-travel representation. We identify four different (sub-)patterns in the representation: a) Before morning commute pattern, which represents the activity-travel undertaken before leaving home to work in the morning, b) Work commute pattern, which represents the activity-travelpursued during the morning and evening commutes, c) Midday pattern, which includes all activity and travel undertaken from work during the midday break, and d) Post home-arrival pattern, which comprises the activity and travelbehavior of individuals after arriving home at the end of the evening commute. The morning and evening commutes are grouped into a single work commute pattern since the travel mode for both these commutes will, in general, be the same. Within each of the before work, midday and post home-arrival patterns in Figure 1, there might be several tours. A tour is a circuit that begins at home and ends at home for the before work and post home-arrivalpatterns and is a circuit that begins at work and ends at work for the midday pattern. Further, each tour within the before work, midday and post home-arrivalpatterns may comprise several activity stops. Similarly, the morning commute and evening commute components of the work commute pattern may also comprise severalactivity stops.

The characterization of the complete workday activity-travel pattern is accomplished by identifying a number ofdifferent attributes within the representation discussed above. These attributes may be classified based on the level of representation they are associated with; that is, whether they are associated with a pattern, a tour, or a stop. Pattern level attributes include the number of tours for the before work, midday and post-home arrival patterns, and the home-stay duration before the morning commute for the work commute pattern. Tour-level attributes include travelmode, number of stops, home-stay duration before each tour in the before work and post home-arrival patterns, work-stay duration before each tour in the midday pattern, and sequence of tour in the pattern. Stop level attributes include activity type, traveltime to stop from previous stop, location of stop, activity duration, and sequence of stop in the tour. 
The analysis of the workday activity travel pattern of individuals entails the modeling of each of the attributes identified in the activity-travelrepresentation. The joint modeling of all the attributes is infeasible because of the large number of attributes and the large number of possible choice alternatives for each attribute. There is a need to develop an analytic framework to model the representation which is feasible to implement from a practical standpoint. The next section descriptively examines the activity-travelpattern of workers using empirical data to inform the process of developing an operational analytic framework.

\section{Descriptive Analysis of Activity-Travel Pattern of Workers}

We use data from two U.S. metropolitan regions in the descriptive analysis to identify consistent patterns that will inform the analytic structure for activity-travel pattern modeling. ${ }^{1}$ The two data sets are obtained from the 1991 Boston Region Household Activity Survey and the 1990 Bay Area Household Travel Survey. Each of the two data sets were subjected to a series of cleaning and screening procedures to select only those individuals who a) went to work on the survey day, b) started their day at home and ended the day at home, c) had no "holes" in their activity-travel pattern on the survey day, and d) had a positive work duration and positive durations for all activity stops.

The 1991 BostonRegionHousehold Activity Survey was conducted by the CentralTransportation Planning Staff (CTPS) of the Boston Metropolitan Planning Organization. The mail-back survey was conducted in April of 1991 and collected data on socio-demographic characteristics of the household and each individual in the household (see Stopher, 1992). The survey also included a one-day (mid-week working day) activity diary to be filled out by all members of the household above five years of age. Each activity pursued by an individual was described by: a) start time, b) stop time, c) location of activity participation, d) travel time from previous activity, e) travel mode to activity location, and f) activity type.

\footnotetext{
${ }^{1}$ The focus here is not on identifying differences in activity-travel patterns across the metropolitan areas. Though such a comparative analysis would be interesting, the emphasis here is on identifying consistency in activity-travel patterns to develop a general operational framework.
} 
The current analysis uses the activity-travel pattern of 2572 individuals who remained in the sample after the cleaning/screening procedures.

The 1990 Bay Area Household TravelSurvey was conducted by the Metropolitan Transportation Commission (MTC) in the Spring and Fall of 1990 (see White and Company, Inc., 1991). This survey included a single-weekday travel diary of individuals, and it is this single-day sample that is used here. The survey also collected data on household and individual socio-demographics. The total sample size in the Bay area data after screening and cleaning is 7559.

In the following descriptive analysis, we will first examine the number of tours in the before work, midday, and post home-arrival patterns. Next, we will examine the number of stops in each pattern, then study interactions in stop-making across different times of the day, and finally examine the interactionin tour mode choice and number of stops in the tour. The findings from the descriptive analysis will form the basis for the analysis framework in Section4.

\subsection{Number of tours}

Table 1 presents the distribution of number of tours in the two data sets for the before work, midday, and pos home-arrival patterns. Two important observations can be made from the table. First, most individuals do not pursue any out-of-home activity before leaving home forwork. On the other hand, about $34 \%$ of individuals in the Boston data (26\% in the Bay area data) pursue activities from work during mid-day and about $32 \%$ of individuals in the Boston data (20\% in the Bay area data) have some form of out-of-home activity involvement after arriving back home from work. Second, almost all individuals who pursue activities during the before work, midday and post home-arrivalpatterns do so in one or two tours (a negligible fraction pursue more than two tours). Also, a majority of individuals make either no tours or one tour.

The second observation made above suggests that we can assume for practical purposes that there are no more than two tours in each of the three patterns. In addition, since most individuals make only one tour in each of the three patterns (if they make any tours), a reasonable modeling strategy would be to focus 
on the presence/absence of a first tour in each pattern (along with the various characteristics of this first tour) and then model the presence/absence of a second tour in the pattern (and the attributes of the second tour) conditionalon the presence and characteristics of the first tour. Further, in accommodating interactions in activity-travel across different periods of the day, it should suffice to confine the interactions to stopmaking in the first tour of the before work, mid-day and post home-arrivalperiods, and stop-making during the morning/evening commute periods (see Section 3.3 for more on the interactions across times of day). That is, we will ignore any interactions between the characteristics of the second tour in each pattern, and the characteristics of the first/second tour of other patterns and stop-making in the morning/evening commute. The basic idea is that any error introduced by these assumptions is going to be small due to the relatively small fraction of individuals making more than one tour in the before work, midday and post home-arrival patterns.

\subsection{Number of stops}

Table 2 presents the number of stops made in the morning/evening commutes, and in the first tour of each of the before work, midday and post home-arrivalpatterns. The results indicate a general trend to making more stops later in the day, especially in the midday, evening commute and post home-arrival periods. The number of stops in the before work pattern is very small. This suggests that we might adopt a modeling strategy where we first focus on number of post home-arrival stops, evening commute stops, and middaystops, next model number of stops in the morning commute pattern conditionalon stop-making at the later times of the day, and finally model number of stops in the before work pattern conditional on stop-making in all other periods. Another observation from the table is that for all periods of the day, the most frequent number of stops is one (if any stops are made at all). Thus, we may model the presence/characteristics of the first stop in each period, next model the characteristics of the second stop in the period conditional on the first (if a person makes two stops), then model the characteristics of the third stop conditional on the characteristics of the first two stops (if a person makes three stops), and so on. 


\subsection{Interaction in stop-making across different times of the day}

In this section, we examine interactions in stop-making in the first midday tour, evening commute, and first post home-arrival tour (as indicated earlier, the midday, evening, and post home-arrival periods represent times of highest activity involvement in the day). We then examine the purpose of activity stops during these three periods.

Table 3 provides the percentage of individuals making one or more stops during each of the three periods controlling for stop-making in other periods. The results indicate that there is little interaction between stop-making in the midday period and the evening commute/post home-arrival periods (note the rather small variation in percentages for the midday column across the 'yes' and 'no' categories for the other two periods and the small variation in the evening commute/post home-arrival columns across the 'yes' and 'no' categories for the midday period). However, there is a substantial substitution effect in stop-making between the evening commute period and the post home-arrival period: individuals making stops during the evening commute are less likely to make post home-arrival stops and vice versa.

To examine the reasons for the nature ofinteractions indicated above, we study the activity purpose of stops during the first midday tour, evening commute, and first post home-arrival tour (Table 4). The Table indicates that $39 \%$ of stops during midday in the Boston data (42\% in the Bay area data) are made for the purpose of eating out. Additionally, about 12\% of stops during midday (about $9 \%$ in the Bay area data) are to return home, presumably also for eating purposes. Thus, about half of all midday stops are for the basic purpose of eating. The other purposes which are engaged in with some frequency are the workrelated and personalbusiness categories. Only a small percentage of stops are made for social-recreational and shopping purposes. In contrast, close to half of all evening commute stops and more than half of all post home-arrivalstops are made for social-recreational and shopping purposes. Further, the eat-out category during the evening commute and post home-arrival periods may also be labeled as a social-recreational activity (in contrast to the more basic biological functionality of the eat-out category during midday). Overall, it appears that since a sizeable fraction of stops during the evening commute and post home-arrival periods are for social-recreational or shopping activities, there is substantial substitution in stop-making 
between these two periods. On the other hand, the stops during the midday serve the more basic function of eating out and work-related tasks. Consequently, the stops are not very substitutable between the midday period and the evening commute/post home-arrival periods.

The modeling implication of the observations made above is that we need to modelnumber of stops in the evening commute and the first post home-arrival tour jointly, but can modelstop-making during the midday period independently.

\subsection{Interaction of mode choice with number of stops}

In general, we might expect that the choice of mode for the work commute is made jointly with the number of stops in the commute. For example, in the Boston data, $38 \%$ of individuals using the drive alone mode for the evening commute made one or more stops during the commute. On the other hand, only $11.7 \%$ of individuals using non-drive alone modes make any evening commute stops. Thus, there appears to be an association between evening commute stop-making and work mode choice. The data also suggests some amount of jointness in work mode choice and number of stops in the first post home-arrival tour. In the Boston data, about $39 \%$ of individuals who use the drive alone mode for the evening commute make a post home-arrival stop compared to $36.7 \%$ of individuals who use other modes. This association (though weak) may be because individuals who want to pursue post home-arrival activities select the drive alone mode to work to enable an early home arrival that will facilitate participation in post home-arrival activities. Similar results are found in the Bay area data.

The above observations, along with those made in section 3.3, suggest a need to modelwork mode choice, number of evening commute stops, and number of stops in the first post home-arrivaltour jointly. However, since the mode chosen for the post home-arrival tours is predominantly the auto mode, we can ignore (for simplicity in modeling) any joint decision-making between number of stops and mode choice in the first post home-arrivaltour and instead model the travelmode for the tour conditionalon stop-making in the tour (note that if the number of stops in the first post home-arrival tour is zero, it implies absence of the tour). 
For each tour in the before work and midday patterns, we propose to model presence/absence of a tour, tour mode choice and number of stops in the tour jointly. The mode choice for the midday tours will be dependent on the mode chosen for the work commute (thus, if an individual uses transit to work, s/he cannot use drive alone for midday tours).

The travel mode for the morning commute is the same as that for the evening commute for most individuals in the sample, but since there exists the possibility that they may be different, we can model mode choice and number of stops for the morning commute conditional on mode choice and number of stops in the evening commute.

\section{Analysis Framework}

Our analysis framework is based on modeling the pattern and tour level attributes first, and then modeling the stop-levelattributes conditionalon the pattern and tour levelattributes. The framework brings together the various observations made from the descriptive analysis in the previous section. Figure 2 shows the framework for analysis of pattern and tour level attributes, and Figure 3 presents the framework for analysis of stop level attributes in each tour for each period. The reader will note that Figures 2 and 3 together capture the entire representation of a worker's daily activity pattern. The number of tours in the before work, midday and post home-arrival patterns, and the sequence of tours in these patterns, are implicitly modeled in Figure 2 by determining if an individual makes a first tour and then conditional on making the first tour, if the individualmakes a second tour (in concept, the procedure can be extended to more than two tours in a pattern, but we restrict the framework here to two tours based on the descriptive analysis of the previous section). Similarly, the sequence of stops in a tour is modeled implicitly in Figure 3 by determining the characteristics of the first stop, then the second conditional on the first, the third conditional on the first two, and so on.

The components of Figure 2 essentially entail two kinds of econometric models. The components labeled (1) in the figure may be modeled using a joint unordered discrete choice and an ordered discrete choice model system (see section 5.1). The components labeled (2) may be modeled using hazard-based 
duration models (see Hamed and Mannering, 1993 and Bhat, 1996 for use of such models to examine activity duration).

The joint activity type choice, activity duration, and travel time duration model labeled (3) in Figure 3 maybe modeled(separately foreach of the periods) using a joint discrete/continuous econometric system (see Bhat, 1998 for the estimation and application of such a joint modelfor the evening commute period). The location choice of the stop (labeled (4) in Figure 3) can be modeled subsequently using disaggregate spatialdestination choice models (see Fotheringham, 1988 and Bhat et al., 1998) by identifying all possible destinations which can be reached by the travelmode assigned for the tour (of which the stop is a part) and within the travel time duration estimated earlier.

The analysis framework proposed above accommodates the spatial-temporalinteractions in stopmaking decisions (since it models activity duration and travel time duration jointly, and then models destination choice based on travel time duration). As indicated by Thill and Horowitz (1997a), little attention has been paid to developing empirical models that explicitly recognize temporal effects on destination choice behavior. Thill and Horowitz (1997b) develop an empiricalmodel of destination choice where the choice set is probabilistically determined based on the distribution of a travel time threshold. While their work is an important step forward in incorporating spatial-temporal interactions in destination choice modeling, their study assumes that there is no prior knowledge of the parameters characterizing the distribution of travel time and that the travel time constraint is not a function of individual attributes (such as income, hours of work, etc.). Our procedure to model travel time jointly with activity duration and as a function of relevant individual attributes provides prior information on the distribution of travel time durationforthe subsequent destination choice set generation. This simplifies the estimationofthe destination choice model while also explicitly incorporating spatial-temporal interaction in stop-making decisions.

\section{Estimation and Application}

The analysis framework presented in the previous section comprises four different types of econometric models. Of these four model types, the structures for the components labeled (2) in Fig. 2 and 
labeled (3) and (4) in Fig. 3 have already been developed and applied, as indicated in the previous section. So, we will not focus on these components in the current paper. Bhat (1997) has developed a model structure for work mode choice and number of commute stops which can be applied to model all the components labeled (1) in Fig. 2, except the joint model of evening commute mode choice, number of evening commute stops, and number of post home-arrivalstops. This joint model has an unordered discrete variable (mode choice) and two ordered discrete variables (evening commute stops and post home-arrival stops), while Bhat's (1997) model is developed for an unordered variable and one ordered variable. In the rest of this paper, we extend Bhat's earlier model to estimate a joint model of evening commute mode, number of evening commute stops, and number of post home-arrival stops.

\subsection{Econometric framework}

In the following presentation of the model structure, we will use the index $i$ to represent mode $(i=1,2, \ldots I)$, index $k$ to represent the number of non-work evening commute stops $(k=0,1,2, \ldots K)$, index $l$ to represent the number of post home-arrival stops $(l=1,2, \ldots L)$, and the index $q$ to represent the $q$ th individual $(q=1,2, \ldots Q)$. The equation system is then as follows:

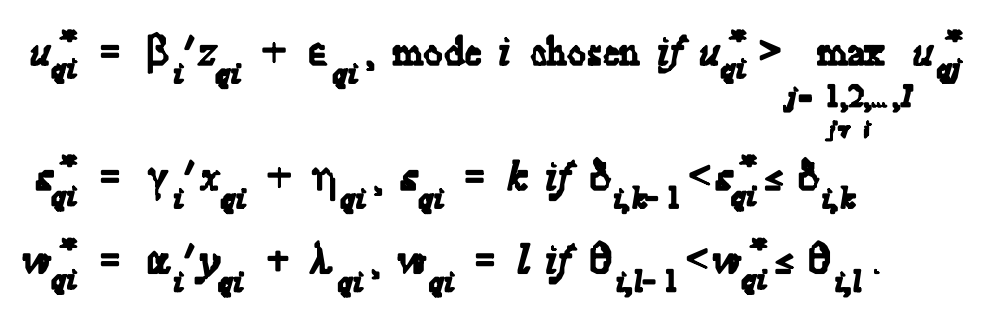

$\mathrm{i} \boldsymbol{u}_{\boldsymbol{q} i}^{*} \mathrm{~s}$ the indirect (latent) utility that the $q$ th individualderives from using the $i$ th mode, $\boldsymbol{s}_{\boldsymbol{q} i}^{*}$ is the (latent) evening commute stop-making propensity ofthe $q$ th individualshould s/he use mode $i, \boldsymbol{s}_{\boldsymbol{e} i}$ is the observed number of evening commute stops conditional on choice of mode $i$ to work ( $\boldsymbol{s}_{\boldsymbol{q} i}$ is unobserved for the non-chosen modes), $\boldsymbol{w}_{\boldsymbol{e} i}^{*}$ is the (latent) post home-arrival stop-making propensity of the $q$ th individual should s/he use mode $i$ for the work commute, and $\boldsymbol{w}_{\boldsymbol{e} i}$ is the observed number of post home-arrival stops if the individual $q$ chooses work mode $i$ ( $\boldsymbol{v}_{\boldsymbol{e} i}$ is unobserved forthe non-chosen work modes). $\boldsymbol{s}_{\boldsymbol{e q}}$ is characterized by the evening commute stop-making propensity $\boldsymbol{s}_{\boldsymbol{q} i}^{*}$ and the threshold bounds (the $\mathbf{8}^{\prime} \boldsymbol{s}$ ) 
in the usual ordered-response fashion. A similar relationship holds between $\boldsymbol{v}_{\boldsymbol{e q}}, \boldsymbol{v}_{\boldsymbol{e q}}^{*}$, and the threshold bounds represented by the $\boldsymbol{\theta}^{\prime} \boldsymbol{s} \cdot \boldsymbol{z}_{\boldsymbol{q} \boldsymbol{i}}, \boldsymbol{x}_{\boldsymbol{e} \boldsymbol{}}$, and $\boldsymbol{y}_{\boldsymbol{q} \boldsymbol{i}}$ are column vectors of exogenous variables, and $\boldsymbol{\beta}_{\boldsymbol{i}}, \boldsymbol{\gamma}_{\boldsymbol{i}}$, and $\boldsymbol{\alpha}_{\boldsymbol{i}}$ are corresponding column vectors of parameters to be estimated. We assume that the $\boldsymbol{E}_{\boldsymbol{q} i}{ }^{\prime} \boldsymbol{s}$ are identically and independently extreme-value distributed (with a location parameter of zero) across alternatives $i$ and individuals $q$. $\eta_{\boldsymbol{q} \boldsymbol{i}}$ and $\boldsymbol{\lambda}_{\boldsymbol{e t}}$ are assumed to be identically (and independently) normal-distributed across individuals $q$ and modes $i$, each with a marginal standard normal distribution function $\Phi($ ).

The ordered-response structure for number of stops in the evening commute and post home-arrival periods explicitly recognizes the ordinal (but discrete) nature of stops. This improves the efficiency of the model system, since substantially fewer parameters need to be estimated in an ordered-response structure compared to an unordered-response structure (Bhat and Pulugurta, 1998). A potential limitation of the ordered-response structure is that it tends to impose a monotonic effect of continuous explanatory variables on stop-making. This issue may be resolved by appropriate variable specification, such as introducing spline effects of the continuous variable, to allow a non-monotonic relationship.

Another possible model structure for number of stops is a count model (such as a Poisson or negative binomial regression). But count models treat the dependent variable as being non-categorical, though taking on only non-negative integer values. If the number of stops has substantial variation (for example, varying from 0 to 75 ), then the count model may be an option. But for the range of stops during the evening commute and post home-arrival periods (between 0 to 4 in the current sample), it is more appropriate to consider stop-making as an intrinsically discrete choice. Thus, the ordered-response structure is better suited for number of stops in the current analysis than a count model.

Let $R_{q i}$ be a dummy variable; $R_{q i}=1$ if the $i$ th mode is chosen by the $q$ th individual forher/his work travel, and $R_{q i}=0$ otherwise. Define

$$
v_{q i}=\left\{\max _{j-1,2, \ldots, j, i+i} u_{* i j}^{*}\right\}-\epsilon_{\alpha i}
$$


The equation system in (1) can now be structured as:

$$
\begin{aligned}
& R_{q i}^{*}=\beta_{i}^{\prime} z_{q i}-v_{q i}, R_{q i}=1 \text { if } R_{q i}^{*}>0, R_{q i}=0 \text { otberwise } \\
& s_{q i}^{*}=\gamma_{i}^{\prime} x_{q i}+\eta_{q i}, s_{q i}=k \text { if } \delta_{i k-1}<s_{q i}^{*} \leq 8_{i k} \\
& w_{q i}^{*}=x_{i}^{\prime} y_{q i}+\lambda_{q i}, w_{q i}=l \text { if } \theta_{i l-1}<w_{q i}^{*} \leq \theta_{i l} .
\end{aligned}
$$

The jointness in the three choices (work mode, number of evening commute stops, and number of posthome arrivalstops) arises because ofpotentialcorrelationamong the randomcomponents $\left(\boldsymbol{v}_{\boldsymbol{e q}}, \boldsymbol{\eta}_{\boldsymbol{q u}} \boldsymbol{\lambda}_{\boldsymbol{q} \mathbf{i}}\right\rangle$. The key to accommodating these correlations is to transform the random variable $v_{\boldsymbol{e i}}$ into a standard normal random variable $\boldsymbol{v}_{\boldsymbol{e} i}^{*}$ as follows:

$$
v_{e i}^{*}=\Phi^{-1}\left[F_{i}\left(v_{q i}\right)\right]
$$

where $\Phi($.$) is the standard normal distribution function and \boldsymbol{F}_{\boldsymbol{i}}$ is the multinomial logit distribution function of $\boldsymbol{v}_{\boldsymbol{q} \boldsymbol{i}}$ implied by equation (2) and the assumed iid extreme value distribution for the $\boldsymbol{E}_{\boldsymbol{q} \boldsymbol{i}} \boldsymbol{s}^{\boldsymbol{s}}$. Now, since $\Phi\left(v_{\boldsymbol{e} i}^{*}\right)=\boldsymbol{F}_{\boldsymbol{i}}\left(\boldsymbol{v}_{\boldsymbol{e} i}\right)$ by construction (see equation 4), we can specify a trivariate distribution $L_{3}$ for $v_{e i}, \eta_{q i}$, and $\lambda_{q i}$ having the marginaldistributions $F_{i}($.$) for v_{q i}$ and $\Phi($.$) for \eta_{q}$ and $\lambda_{q}$, as (Lee, 1983):

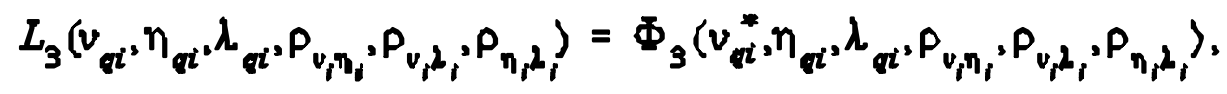

where $\Phi_{3}($.) denotes the trivariate normal distribution. From equation (3) and equation (5), the joint probability of choosing mode $i$, number of evening commute stops $k$, and number of post-home arrival stops $l$ for individual $q$ is: 


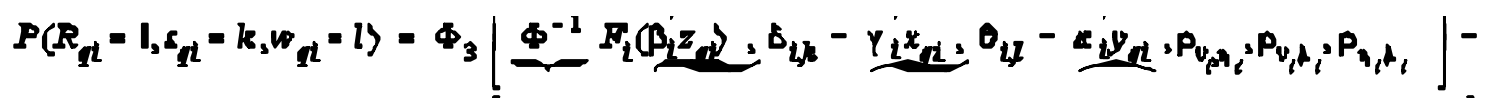

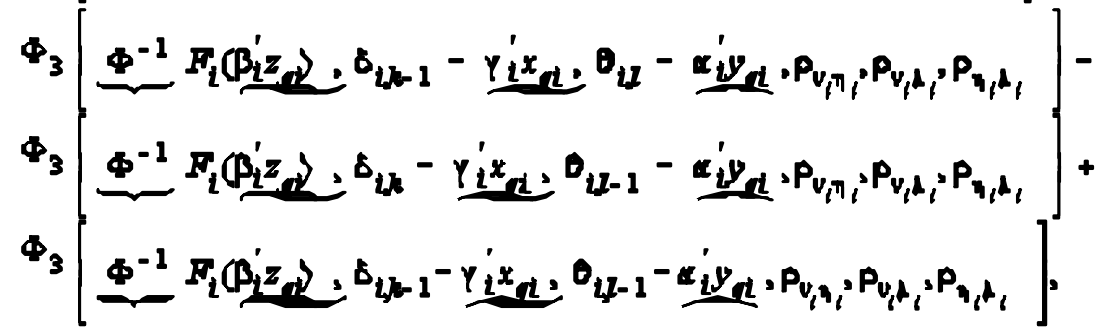

where

$$
F_{i}\left(\beta_{i}^{\prime} z_{q i}\right)=\operatorname{Prob}\left(v_{e i}<\beta_{i}^{\prime} z_{e i}\right)=\frac{\exp \left(\beta_{i}^{\prime} z_{e i}\right)}{\sum_{j=1}^{I} \exp \left(\beta_{j}^{\prime} z_{e j}\right)}, i=1,2, \ldots I .
$$

The parameters to be estimated in the joint model are the $(K-1) \mathbf{8}_{\boldsymbol{i} \boldsymbol{k} \mathbf{k}}$ parameters $\left(\boldsymbol{\delta}_{\boldsymbol{i} \mathbf{O}}=-\infty, \boldsymbol{\delta}_{\boldsymbol{i} \boldsymbol{I}}=+\infty\right)$, the $(L-1) \boldsymbol{\theta}_{\boldsymbol{\zeta l}}$ parameters $\left(\boldsymbol{\theta}_{\boldsymbol{\zeta} \mathbf{O}}=-\infty, \boldsymbol{\theta}_{\boldsymbol{\zeta I}}=+\infty\right)$, and the

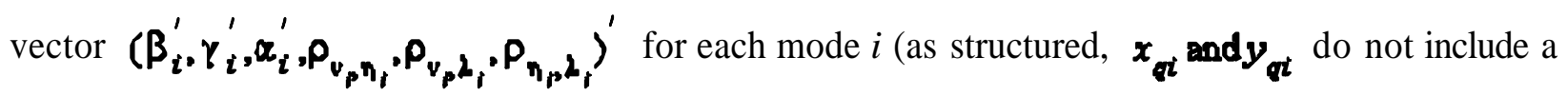
constant). Defining a set of dummy variables

$$
M_{\text {eld }}=\left\{\begin{array}{l}
1 \text { if individual } q \text { makes } k \text { evening stops and } l \text { post-home arrival stops } \\
0 \text { otherwise. } \\
(q=1,2, \ldots, k=1,2, \ldots, l=1,2, \ldots L)
\end{array}\right.
$$

the log likelihood function for the estimation of the parameters in the model takes the form

$$
\log \mathscr{L}=\sum_{q=1}^{\Omega} \sum_{i=1}^{l}\left\{R_{\alpha i}\left(\sum_{k=1}^{L} \sum_{l=1}^{L} M_{q d i} \log \left[P\left(R_{q i}=1, s_{q i}=k, v_{q i}=l\right)\right]\right) .\right.
$$

\subsection{Data source and sample}

The data source used here is the household activity surveyconducted by the Central Transportation Planning Staff (CTPS) in the Boston Metropolitan region. The sample comprises 1440 employed adult individuals who made a work-trip on the diary day and were older than 16 years (complete details of the screening and data cleaning procedures employed in arriving at this sample from the overall activity diary 
data is provided in Singh, 1997). The mode choice estimation involves three modes: drive alone (use of a car/van/pickup truck by one traveler), shared ride (use of a car/van/pickup truck by more than one traveler) and transit (bus, commuter rail, or local rail). The travel mode used for the first leg from work in the evening is used as the work mode choice. Thus, if a person picks up another family member or a child by car at an intermediate point in the evening commute and then proceeds to home, the person's work mode choice is classified as drive alone. Picking up individuals during the evening commute is included as a stop; however, if an individual rides with another person from the work place and drops the person at an intermediate point during her/his commute, the work mode assigned to the individualis shared ride and the "drop-off" is not recognized as a stop. The number of stops in the sample varies between 0 and 4 for both the evening commute and post home-arrival stops.

\subsection{Model specification and overall data fit}

The choice of variables for potentialinclusion in the model was guided by previous empirical work on mode choice modeling and trip chaining analysis, intuitive arguments regarding the effects of exogenous variables, and available data. We arrived at the final specification based on statistical testing of alternative specifications.

We maintained equalparameters on all exogenous variables across the different mode regimes for the evening commute and post home-arrival propensity equations. We further restricted the thresholds in each stop-making propensity equation to be the same for each mode regime up to a structural shift (that is, all thresholds for each mode regime are shifted by the same amount relative to the corresponding threshold in a base mode regime; the total number of parameters characterizing the thresholds in this specification is six in each stop-making equation, fourthresholds in the base mode regime and two structural

shift terms for the two remaining mode regimes). This specification is equivalent to maintaining the same thresholds for all mode regimes, but introducing constants in the stop equations in two of the three mode regimes. 
Finally, we constrained the correlation in unobserved factors affecting the evening commute and post home-arrival stop-making propensities to be equal across the mode regimes (i.e., $P_{n_{i}, i}=P_{n, 2} \forall \boldsymbol{i}$. ). The correlations between unobserved factors affecting the propensity to choose the shared ride/transit modes to work and the post home-arrival stop-making propensity were found to be statistically insignificant. Consequently, these correlation terms were dropped. The unobserved correlation in mode choice and evening stop propensity was not statistically different between the shared-ride and transit regimes and so these two correlations were constrained to be the equal. There are four correlation parameters in the final specification representing correlation in unobserved factors affecting a) drive alone utility and evening stop-making propensity, b) shared ride/transit utility and evening stop-making propensity, c) drive alone utility and post home-arrivalstop-making propensity, and d) evening stop-making and posthome arrival stop-making propensities (same across all mode regimes).

The log-likelihood value at convergence for the joint model system is -3437.09. The log-likelihood when only alternative specific constants are included in the mode choice modeland when only the threshold parameters are introduced in the number of stops model (with all correlation parameters set to zero) is 3881.05. A log-likelihood ratio test clearly rejects the null hypothesis that all exogenous variable parameters and error correlations are zero. A further test of the joint model with an independent model (where all the correlation terms are set to zero) rejects the hypothesis that mode choice, number of evening commute stops, and number of post home-arrival stops are independently determined (the log-likelihood value of the independent model is $-\mathbf{3 4 5 6 . 5 6}$; the likelihood ratio value for the test is 38.94 which is larger than the chi-squared statistic with four degrees of freedom at any reasonable level of significance).

\subsection{Parameter estimates}

The effects of all the variables on mode choice are provided in Table 5 . The results are selfexplanatory. The implied cost of in-vehicle travel time is $\$ 6.21$ per hour and that of out-of-vehicle time is $\$ 8.10$ per hour (computed at the mean one-way travel distance of 9.36 miles).

The effect of exogenous variables on evening commute and post home-arrival stop-making propensity are shown in Table 6 . The positive influence of income on evening commute stop-making 
propensity is consistent with the results from earlier studies (see Strathman et al., 1994; Goulias and Kitamura, 1989), as is the higher stop-making propensity of married women compared to men (see Mensah, 1995). Both these variables, however, do not impact post home-arrival stop-making propensity. On the other hand, age has a negative impact on post home-arrival stops but no statistically significant effect on commute stop-making. Individuals in households with young children make less evening commute and post home-arrival stops, possibly because they are faced with the responsibility of taking care of young children at home. Interestingly, however, individuals in households with children aged 12-16 years have a high propensity to make post home-arrival stops. This result may be a combination of two factors. First, older children require less attention and supervision compared to younger children, thus allowing adults in the household to pursue out-of-home activities. Second, the recreational desires of older children may encourage the entire family to participate in out-of-home activity. The number of adults in a household has a highly significant negative influence on evening commute stop-making propensity: the greater the number of adults, the more opportunity there is to share the responsibility of household maintenance activities. The finalsocio-demographic variable affecting stop-making is associated with household structure. The effects of the work schedule and level-of-service variables in Table 6 appear to capture time availability constraints; individuals who work more, who leave work after $6 \mathrm{pm}$, and who spend more time in travel to work have less time available for post-work activities, and so engage less in such activities. The threshold values relate the stop-making propensity to the observed number of stops.

The joint modeling of mode choice, number of stops during the evening commute, and number of stops after arriving home from work is necessitated by the potential presence of correlation in unobserved elements affecting the three decisions. The results (see Table 7) indicate statistically significant correlations.

The parameter estimates in the first and second rows of Table 7 represent the correlation between the error terms $v_{\boldsymbol{e} i}$ and $\eta_{\boldsymbol{q} i}$ for the drive alone mode and shared-ride/transit modes, respectively (see equation 3). Since the error term $v_{\boldsymbol{e} i}$ enters the mode choice utility equation negatively, a positive parameter in the first two rows of Table 7 actually indicates a negative correlation between unobserved factors affecting the corresponding mode utility and evening stop-making propensity, while a negative parameter implies a positive correlation. A similar result holds for the correlation in unobserved factors 
between the drive alone mode utility and the post home-arrivalstop-making propensity in the third row of the table. The results show that unobserved factors (say, need forcontroland independence, impulsiveness, high mobility, etc.) that increase the preference for the drive alone mode also increase stop-making propensity during the evening commute and the post-home arrivalperiods. On the other hand, unobserved factors (say, low activity levels) that increase the preference for the shared-ride and transit modes decrease evening commute stop-making propensity. Thus, the choice of mode is not exogenous to stop-making; individuals who would like to make more evening commute stops and more post-home arrival stops tend to choose the drive alone mode to work, all observed characteristics being equal. The last row of Table 7 shows a negative association in unobserved factors influencing evening stop-making and post homearrival stop-making, re-inforcing the discussion in section 3.3 regarding substitution effects in stop-making between these two periods.

As we will see in the next section, ignoring the correlations in Table 7 have substantialimplications for policy analysis.

\subsection{Policy implications}

In this section, we examine the impacts of changes in policy-relevant exogenous variables on the number of stops by the drive alone mode during the evening commute and total number of stops during the post home-arrival period. We confine our attention to the impact on stops made by the drive alone mode

for the evening commute because drive alone stops contribute most to traffic congestion (on the other hand, transit stops do not contribute to vehicle-trips since transit service is independent of whether an individual using transit decides to make a stop or not). Almost all stops made after arriving home are made by the auto mode and so we compute the effect on total post home-arrival stops (independent of the work mode used).

The impact of policy actions on stop-making can be evaluated by modifying exogenous variables to reflect a change, computing revised disaggregate probabilities for each "evening stop-post home-arrival stop"combination by the drive alone mode to work, summing the disaggregate probabilities across all posthome arrivalstop categories for each evening stop category, computing revised expected aggregate values 
for number of evening stops by the drive alone mode, and then obtaining a percentage change from the baseline estimates. The effect of policy actions on total post-home arrival stops is obtained by modifying exogenous variables to reflect a change, computing revised disaggregate probabilities for each "work travel mode-evening stop-post home arrival stop" combination, summing the disaggregate probabilities across all evening stop categories and work travel modes for each post home-arrival stop category, computing revised expected aggregate values for number of total post home-arrival stops, and then obtaining a percentage change from the baseline estimates.

We compare the estimated impact of policy actions from the joint model proposed in this paper and an independent model system that involves the estimation of independent mode choice and number of stops models. Table 8 provides the estimated percentage change (at the aggregate level) in evening stops by the drive alone mode and in (total) post home-arrival stops in response to transit service improvements and an increase in auto-use costs. The transit service improvements involve a five minute decrease (on average across the sample) in transit in-vehicle and out-of-vehicle travel times (note that since some individuals have a current in-vehicle/out-of-vehicle time of less than five minutes, it is not possible to decrease traveltime by five minutes on an individualbasis and hence the scenario of a five minute decrease on average). The five minute decrease (on average) corresponds to about a $17.7 \%(22 \%)$ decrease in transit in-vehicle (out-of-vehicle) time for each individual in the sample. The increase in auto cost involves a 50 cents hike (on average across the sample) in drive alone costs to work. This hike corresponds to a $26.9 \%$ increase in drive alone cost for each individual in the sample (in the rest of this section, we will refer to evening commute stops by the drive alone mode as "evening stops" for brevity).

The results in Table 8 indicate that the independent model shows an almost equal percentage decrease across evening stop categories for all the congestion-alleviation policy measures. However, the joint model suggests that individuals who make many evening commute stops are unlikely to be drawn away from the drive alone mode. This is a consequence of the significant positive correlation between the soloauto utility and evening stop-making propensity.

The results for the post home-arrival stops in response to the transit service improvements show that the independent model underestimates (relative to the joint model) the increase in post home-arrival 
stops . This is because of a combination of several(not easily disentangled) reasons. First, the draw away from the drive alone mode to work due to the transit service improvements is largest for individuals who make fewer post home-arrivalstops and smallest for individuals who make many post home-arrival stops (for example, the draw away from the drive alone mode to work due to the improvement in transit out-ofvehicle time was in the range of 1.74-1.98\% for all the post home-arrival stop categories in the independent model; the corresponding draw values varied much more from $2.24 \%$ for individuals making no post homearrival stops to $1.14 \%$ for individuals making 4 stops in the joint model). The uneven draw in the joint modelis due to the positive correlation in unobserved factors between the drive alone utility and post homearrival stop-making propensity. Now, those who "switch" to transit from the drive alone mode make fewer post home-arrival stops because the travel time to work tends to be higher after the "switch". Since the "switchers" are evenly drawn from all post home-arrival stop categories in the independent model, but drawn most from people making fewer post home-arrivalstops in the joint model, the net result is that there is an under-estimation in total post-home arrival stop-making by the independent model. Second, those who "switch" from drive alone to transit as the work mode are much less likely to make evening commute stops by the specification of the joint model because of the negative correlation in unobserved factors influencing transit use and number of evening commute stops. The decreased number ofevening commute stops of these "switchers" leads to more post home-arrival stop-making due to the negative correlation (or substitution effect) between evening commute stop-making propensity and post home-arrivalstop-making propensity. By ignoring the correlation effects just discussed, the independent model again under-estimates post home-arrival stops. Third, for transit users, the decrease in transit travel time to work implies more time availability and so more post home-arrival stop-making (this effect is almost the same in both the independent model and the joint model, since the coefficient on traveltime to work in the post home-arrival stops model was found to be about the same in both models). The combination of the three effects discussed above leads to a net increase in post home-arrivalstops in both the independent and joint model, but an under-estimation of the increase by the independent model.

The underestimation in the change in post home-arrival stops in response to a congestion pricing strategy (i.e., increase in drive alone cost to work) by the independent modelcan be explained in a similar 
manner as for the transit service improvements. The only difference is that the third effect is not present because travel cost to work does not affect post home-arrival stop-making propensity. Consequently, the independent model predicts (incorrectly) that there is an overall decrease in post home-arrival stops.

The net percentage change in evening commute stops and post home-arrival stops can be computed as:

$$
\text { Net } \% \text { ohange in stops }=\sum_{k=0}^{\sum_{-1}^{4}}\left(\frac{k n_{k}}{\sum_{k} k n_{k}}\right) \zeta_{k}
$$

where $\boldsymbol{n}_{\boldsymbol{k}}$ is the expected number of individuals who make $\mathrm{k}$ evening commute (or k post home- arrival) stops before implementation of the policy action, and $\boldsymbol{\zeta}_{\boldsymbol{k}}$ is the percentage aggregate change in each stop category. This overall effect on number of stops is shown in the column labeled "Net Effect". The independent model projects a substantially more optimistic view of the reduction in evening commute stops than the joint model; in particular, the independent modeloverestimates the percentage reduction in evening stops by more than $50 \%$ for every policy scenario. The independent modelalso underestimates the increase in post-home arrivalstops in response to transit service improvements to work by $36 \%$ fortransit in-vehicle time and by $84 \%$ for transit out-of-vehicle time. As discussed earlier, the direction of the change in posthome arrival stops predicted by the independent model is itself incorrect in response to an increase in drive alone cost to work.

The net effect on the sum of evening commute stops and post-home arrival stops (or total postwork stops) is provided in the final column of Table 8 . The figures in this column show that use of the independent model overestimates the decrease in total post-work stops due to congestion alleviation actions by $80 \%$ to $109 \%$. Such substantialoverestimates of the benefits of traffic controlmeasures can lead to misdirected policy actions and underscores the need to modelwork mode choice and non-work activity stop-making jointly. The results are also important from a mobile-source emissions standpoint, since the independent model overestimates the reduction in total vehicle stops in response to Transportation Control Measures (TCMs). 


\section{Summary and Conclusions}

This paper develops a representation for the workday activity-travel pattern of an individual and presents an operational analytic framework for the representation. The analytic framework treats time as a continuous variable and accommodates spatial-temporalinteractions in activity-travel decisions. The unit of analysis in the framework includes pattern level, tour-level, and stop-level attributes to capture the activity-travel pattern of workers in a comprehensive manner.

The analysis structure in the activity-travelgeneration model system involves the decomposition of the activity-travel pattern representation into several modeling components with sequentiality maintained among the modeling components. This decomposition is based on empiricalobservations of activity-travel patterns which ensures that the sequential structure of model components, even if inappropriate, will produce little error in modeling accuracy. Each of the components involve the joint modeling of several attributes. The econometric structure for the modeling components have been formulated and applied earlier, except for the joint model of evening commute work mode, number of evening commute stops, and number of post home-arrival stops. The current paper formulates a structure for this joint model. The methodology developed here represents, to the authors' knowledge, the first attempt in discrete choice literature to formulate and estimate a model with an unordered multinomialchoice as well as two ordered multinomial choices.

The empirical analysis uses a data set from the Boston Metropolitan area to estimate the relevant parameters of the joint model of evening commute work mode, number of evening commute stops, and number of post home-arrival stops. The results indicate the strong effects of socio-economic variables, residential/workplace locationcharacteristics, work schedule characteristics, and level-of-service measures on evening commute mode choice and number of stops during the evening commute/post home-arrival periods. Our empirical analysis also shows strong correlations in random components among these three choice decisions. The correlations have a substantialimpact on the aggregate percentage change in number of stops during the evening commute and post home-arrival periods due to changes in policy-relevant exogenous variables. In general, ignoring the correlations leads to a rather substantial overestimate of the 
decrease in stop-making after work. This can lead to misdirected policy actions for traffic congestion alleviation and for mobile-source emissions reduction.

\section{Acknowledgements}

This research was funded in part by National Science Foundation grants DMS 9208758 and DMS 9313013 to the National Institute of Statistical Sciences (NISS). It was also funded in part by the US Department of Transportation through the Region One University Transportation Center. The authors would like to thank Mr. Jim Gallagher and Mr. Ian Harrington of the Central Planning Transportation Staff in Boston for providing the data and for clarifying data issues. Thanks are also due to Jay Carini for help with the figures. 


\section{References}

Bhat, C.R. (1996) A hazard-based duration model of shopping activity with nonparametric baseline specification and nonparametric controlfor unobserved heterogeneity, Transportation Research, 30B, 189-207.

Bhat, C.R. (1997), Work travel mode choice and number of nonwork commute stops, Transportation Research, 31B, 41-54.

Bhat, C.R. (1998). Modeling the commute activity-travel pattern of workers: formulation and empirical analysis, technical paper, Department of Civil Engineering, UT Austin.

Bhat, C.R. and F.S. Koppelman (1993) An endogenous switching simultaneous equation system of employment, income and car ownership, Transportation Research, 27A, 447-459.

Bhat, C.R., A. Govindarajan and V. Pulugurta (1998) Disaggregate attraction-end choice modeling: formulation and analysis, forthcoming, Transportation Research Record.

Bhat, C.R. and V. Pulugurta (1998) A comparative analysis of two alternative choice mechanisms for household auto ownership decisions, Transportation Research, 32B, 10-24.

Damm, D. (1980) Interdependencies in activity behavior", Transportation Research Record, 750, 33-40.

Fotheringham, A.S. (1988). Customer store choice and choice set definition, Marketing Science, 7, 299310.

Gärling, T., M.P. Kwan and R.G. Golledge (1994) Computational-process modeling of household travel activity scheduling", Transportation Research, 25B, 355-364.

Goulias, K.G. and R. Kitamura (1989). Recursive model system for trip generation and trip chaining, Transportation Research Record, 1236, 59-66.

Hamed, M.M and F.L. Mannering (1993), Modeling travelers' postwork activity involvement: toward a new methodology", Transportation Science, 27, 381-394.

Hivert, L., M.S. Massot, J.P. Orfueil and P.Troulay (1987). "The Analysis of Present and Future Mobility: New Challenges, New Paradigms", Presented at the Fifth International Conference on Travel Behavior, Aix-en-Provence, France.

Hoorn, T. van der (1983). "Development of an Activity Model Using a One-Week Activity-Diary Data Base", in S. Carpenter \& P. Jones (eds.), Recent Advances in TravelDemand Analysis, 335-349, Gower, Aldershot, England.

Lee, L.F. (1983). Generalized econometric models with selectivity, Econometrica, 51, 507-512.

Mensah, J. (1995). Journey to work and job characteristics of the urban poor: A gender analysis of a survey data from Edmonton, Alberta, Transportation, 22, 1-19.

Singh, S.K. (1997) A joint model of work travel mode choice and number of non-work activity stops, Master's Thesis, Department of Civil and EnvironmentalEngineering, University of Massachusetts, Amherst. 
Stopher, P.R. (1992) Use of an activity-based diary to collect household traveldata, Transportation, 19, 159-176.

Thill, J-C. and J.L. Horowitz (1997a), Travel time constraints on destination-choice sets, Geographic Analysis, 29, 108-123.

Thill, J-C. and J.L. Horowitz (1997b), Modeling nonwork destination choices with choice sets defined by travel time constraints, Department of Geography, State University of New York at Buffalo, Working Paper.

White, E.H. and Company, Inc. (1991) 1990 Bay Area Travel Survey: Final Report, submitted to the Metropolitan Transportation Commission, Oakland, California. 

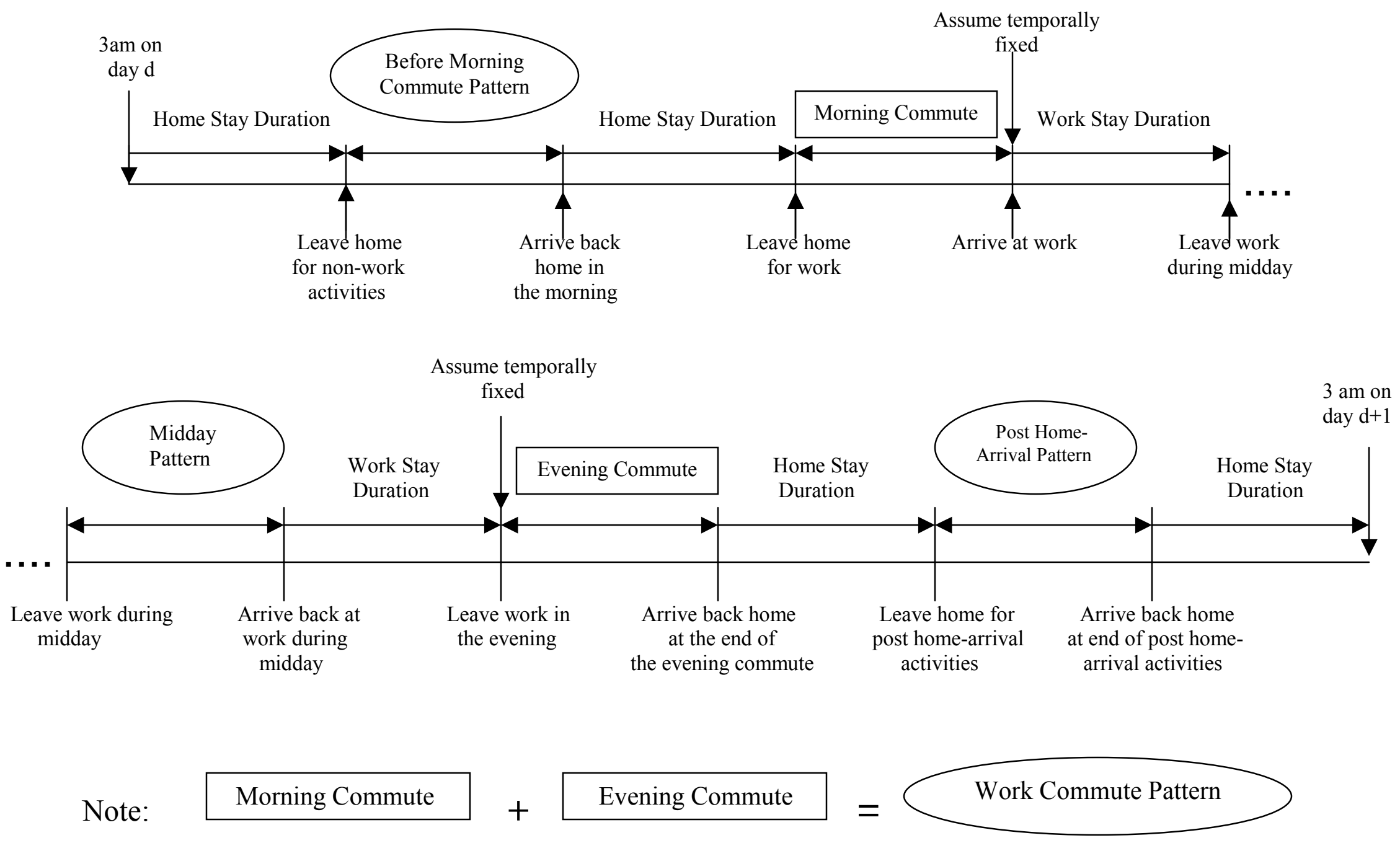

Figure 1. Diagrammatic representation of a worker's daily activity travel pattern 


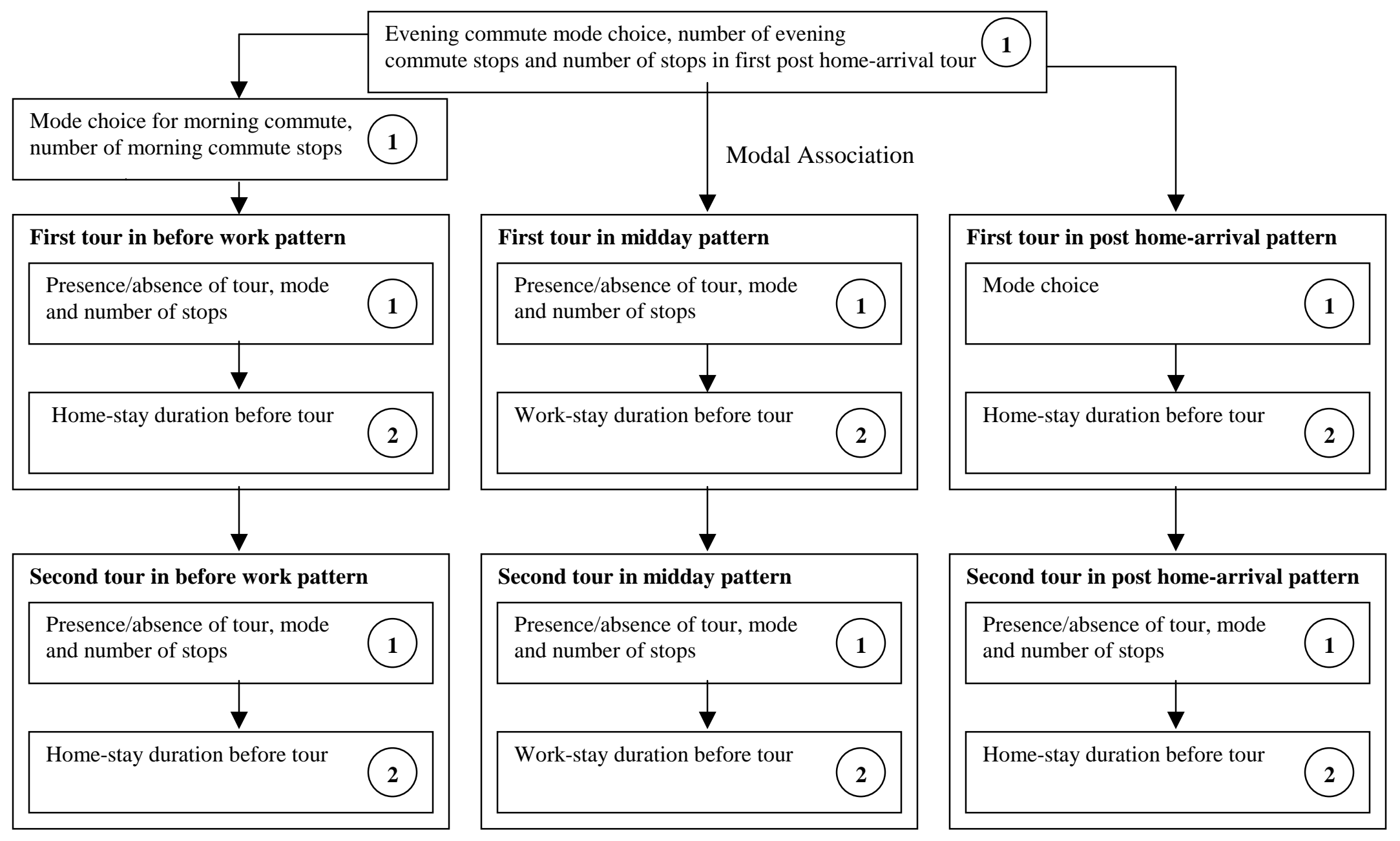

Note: The components in the figure labeled labeled 2 may be modeled using a hazard duration model.

(1) may be modeled using a joint unordered-ordered discrete choice system. The components

Figure 2. Analysis framework for pattern and tour-level attributes 


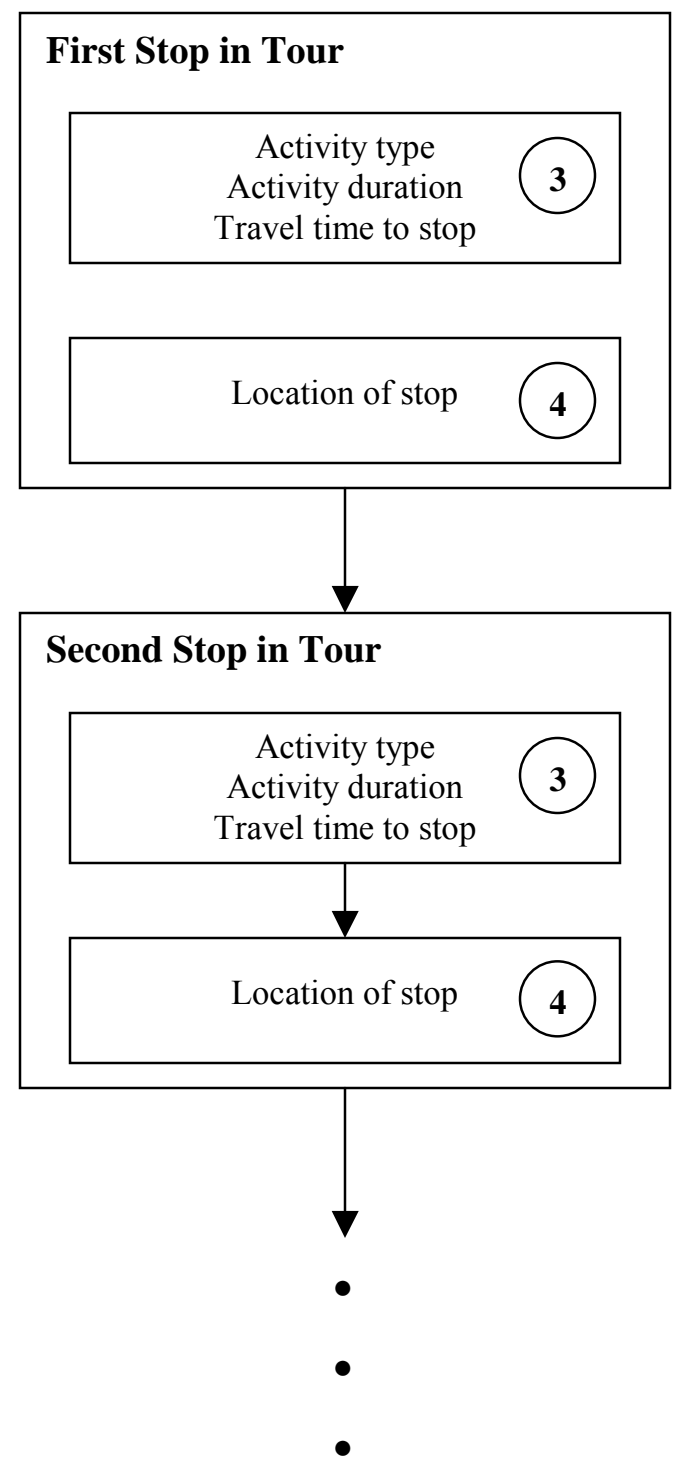

Figure 3. Analysis framework for stop-level attributes of workers 
Table 1. Distribution of Number of Tours

Boston data (Bay area data)

\begin{tabular}{||c|c|c|c||}
\hline \multirow{2}{*}{ \# of tours } & \multicolumn{3}{|c||}{ Percentage of each number of tours in... } \\
\cline { 2 - 4 } & Before work pattern & Midday pattern & $\begin{array}{c}\text { Post home-arrival } \\
\text { pattern }\end{array}$ \\
\hline 0 & $96.9(96.9)$ & $65.6(74.0)$ & $67.6(79.7)$ \\
\hline 1 & $3.0(2.9)$ & $29.2(22.5)$ & $28.5(18.1)$ \\
\hline 2 & $0.1(0.2)$ & $4.0(2.8)$ & $3.4(1.9)$ \\
\hline$? 3$ & $0.0(0.0)$ & $1.2(0.7)$ & $0.5(0.3)$ \\
\hline
\end{tabular}

Table 2. Distribution of Number of Stops

Boston data (bay area data)

\begin{tabular}{||c|c|c|c|c|c||}
\hline \multirow{2}{*}{ \# of stops } & \multicolumn{5}{|c|}{ Percentage of each number of stops in... } \\
\cline { 2 - 7 } & $\begin{array}{c}\text { Before work } \\
\text { pattern }\end{array}$ & $\begin{array}{c}\text { Morning } \\
\text { commute }\end{array}$ & $\begin{array}{c}\text { Midday } \\
\text { pattern }\end{array}$ & $\begin{array}{c}\text { Evening } \\
\text { commute }\end{array}$ & $\begin{array}{c}\text { Post home- } \\
\text { arrival pattern }\end{array}$ \\
\hline 0 & $96.9(96.9)$ & $85.3(85.2)$ & $65.6(74.0)$ & $64.8(74.0)$ & $67.6(79.7)$ \\
\hline 1 & $2.3(2.2)$ & $11.3(11.5)$ & $24.6(17.8)$ & $24.1(17.8)$ & $23.1(14.2)$ \\
\hline 2 & $0.5(0.5)$ & $2.8(2.5)$ & $6.4(4.4)$ & $7.9(5.1)$ & $6.8(3.9)$ \\
\hline 3 & $0.2(0.3)$ & $0.5(0.6)$ & $2.4(2.0)$ & $2.4(1.9)$ & $2.0(1.4)$ \\
\hline 4 & $0.1(0.1)$ & $0.1(0.2)$ & $0.5(0.9)$ & $0.7(0.9)$ & $0.5(0.5)$ \\
\hline$? 5$ & $0.0(0.0)$ & $0.0(0.0)$ & $0.5(0.9)$ & $0.1(0.3)$ & $0.0(0.3)$ \\
\hline
\end{tabular}


Table 3. Interaction in Stop-Making Across Different Times of Day

Boston data (Bay area data)

\begin{tabular}{||l|c|c|c|c||}
\hline \multirow{2}{*}{ Control Variable } & \multirow{2}{*}{ Value Label } & \multicolumn{3}{|c|}{ Percentage of individuals making a stop during... } \\
\cline { 3 - 5 } & & Midday & Evening commute & $\begin{array}{c}\text { Post home- } \\
\text { arrival period }\end{array}$ \\
\cline { 3 - 5 } $\begin{array}{l}\text { Made a mid-day } \\
\text { stop? }\end{array}$ & Yes & - & $36.7(28.0)$ & $32.8(23.2)$ \\
\hline No & - & $34.4(25.3)$ & $32.2(20.5)$ \\
\hline $\begin{array}{l}\text { Made an evening } \\
\text { commute stop? }\end{array}$ & Yes & $35.9(27.6)$ & - & $25.3(13.2)$ \\
\hline $\begin{array}{l}\text { Made a post-home } \\
\text { arrival stop? }\end{array}$ & Yes & $33.6(25.4)$ & - & $36.3(22.8)$ \\
\hline
\end{tabular}

Table 4. Distribution of Activity Type of Stops

Boston data (Bay area data)

\begin{tabular}{||c|c|c|c||}
\hline \multirow{2}{*}{ Activity type } & \multicolumn{3}{|c|}{ Percentage of stops for each activity type during... } \\
\cline { 2 - 4 } & Midday & Evening commute & $\begin{array}{c}\text { Post home-arrival } \\
\text { period }\end{array}$ \\
\hline Home & $11.6(8.9)$ & $0.0(0.0)$ & $0.0(0.0)$ \\
\hline Pick-up/drop off & $1.5(2.8)$ & $10.9(14.4)$ & $11.7(11.0)$ \\
\hline Work-related & $18.4(19.3)$ & $2.6(10.1)$ & $1.8(2.8)$ \\
\hline School & $1.5(0.3)$ & $1.6(2.3)$ & $1.8(4.2)$ \\
\hline Shopping & $9.6(5.5)$ & $30.1(27.2)$ & $25.2(24.3)$ \\
\hline Social/recreational & $2.9(3.7)$ & $16.5(15.6)$ & $32.8(27.3)$ \\
\hline Eat-out & $39.1(41.9)$ & $7.0(4.8)$ & $11.0(12.4)$ \\
\hline Personal Business & $15.4(13.5)$ & $31.3(25.6)$ & $15.7(15.7)$ \\
\hline
\end{tabular}


Table 5. Mode Choice Model Estimates

\begin{tabular}{|c|c|c|}
\hline Variable & Coefficient & t-statistic \\
\hline \multicolumn{3}{|l|}{ Mode constants (drive alone is base) } \\
\hline Shared-ride & -1.117 & -3.12 \\
\hline Transit & 1.408 & 3.42 \\
\hline \multicolumn{3}{|l|}{ Socio-demographic characteristics } \\
\hline \multicolumn{3}{|l|}{ Income - in $\$ 0000 / y r$} \\
\hline Drive alone & 0.118 & 4.60 \\
\hline \multicolumn{3}{|l|}{ Vehicles per worker in household } \\
\hline Drive alone & 1.044 & 5.90 \\
\hline \multicolumn{3}{|l|}{ Number of adults in household } \\
\hline Shared ride & 0.254 & 3.00 \\
\hline \multicolumn{3}{|l|}{ Location characteristics } \\
\hline \multicolumn{3}{|l|}{ Population density in household zone } \\
\hline Drive alone & -0.098 & -1.55 \\
\hline \multicolumn{3}{|l|}{ Employment density at work zone } \\
\hline Transit & 0.029 & 6.29 \\
\hline \multicolumn{3}{|l|}{ Work schedule characteristics } \\
\hline \multicolumn{3}{|l|}{ Arrival at work before 8 am } \\
\hline Transit & -0.656 & -3.03 \\
\hline \multicolumn{3}{|l|}{ Departure between 4 and $6 \mathrm{pm}$} \\
\hline Shared ride & 0.274 & 1.52 \\
\hline \multicolumn{3}{|l|}{ Level of Service Measures (generic) } \\
\hline Total travel time to work - in minutes & -0.054 & -6.54 \\
\hline Out-of-vehicle travel time over distance to work - in mins/mile & -0.336 & -5.62 \\
\hline Total travel cost to work - in dollars & -0.442 & -3.06 \\
\hline
\end{tabular}


Table 6. Number of Stops (in Work Commute) Model

\begin{tabular}{|c|c|c|c|c|}
\hline \multirow[t]{2}{*}{ Variable } & \multicolumn{2}{|c|}{$\begin{array}{l}\text { Evening commute stop } \\
\text { propensity }^{1}\end{array}$} & \multicolumn{2}{|c|}{ Post home-arrival stop propensity } \\
\hline & Coefficient & t-stat. & Coefficient & t-stat. \\
\hline \multicolumn{5}{|l|}{ Socio-economic characteristics } \\
\hline Income - in $\$ 0000 / y r$ & 0.035 & 2.83 & - & - \\
\hline Female and married & 0.241 & 3.36 & - & - \\
\hline Age & - & - & -0.055 & -2.17 \\
\hline Presence of kids $<12 \mathrm{yrs}$ & -0.168 & -2.19 & -0.178 & -2.43 \\
\hline Presence of kids $12-16$ yrs & - & - & 0.524 & 6.90 \\
\hline Number of adults in household & -0.204 & -5.76 & - & - \\
\hline Single parent households & 0.599 & 2.07 & -0.412 & -1.22 \\
\hline \multicolumn{5}{|l|}{ Work schedule characteristics } \\
\hline Work duration (in $10^{-2}$ minutes) & -0.107 & -3.25 & -0.116 & -3.66 \\
\hline Departure before $4 \mathrm{pm}$ & 0.598 & 3.97 & 0.962 & 6.59 \\
\hline Departure between 4 and $6 \mathrm{pm}$ & 0.461 & 3.59 & 0.559 & 4.47 \\
\hline \multicolumn{5}{|l|}{ Level of Service Measures } \\
\hline Total travel time to work - in minutes & -0.004 & -1.96 & -0.004 & -1.822 \\
\hline Out-of-vehicle time over distance - in mins/mile & -0.030 & -2.45 & - & - \\
\hline \multicolumn{5}{|l|}{ Threshold Propensity demarcating } \\
\hline zero and one stop & 0.016 & 0.07 & 0.098 & 0.40 \\
\hline one and two stops & 0.840 & 3.36 & 0.950 & 3.89 \\
\hline two and three stops & 1.396 & 5.48 & 1.619 & 6.37 \\
\hline three and four stops & 1.871 & 7.16 & 2.186 & 8.29 \\
\hline
\end{tabular}


Table 7. Estimates of Correlation

\begin{tabular}{||l|c|c||}
\hline \multicolumn{1}{|c|}{ Correlation in error components between... } & Estimate & t-statistic \\
\hline \hline Drive alone utility and evening stop-making propensity equations & -0.4233 & -2.71 \\
Shared-ride/Transit utility and evening stop-making propensity & 0.1503 & 2.24 \\
Drive alone utility and post-home arrival stop-making propensity & -0.2112 & -1.92 \\
Evening and post-home arrival stop making propensities & -0.2819 & -5.26 \\
\hline
\end{tabular}


Table 8: Impact of Policy Actions

\begin{tabular}{|c|c|c|c|c|c|c|c|c|c|}
\hline \multirow[b]{2}{*}{ Policy Scenario } & \multirow[b]{2}{*}{ Model } & \multirow{2}{*}{$\begin{array}{l}\text { Stop } \\
\text { Category }\end{array}$} & \multicolumn{7}{|c|}{ Percentage aggregate change } \\
\hline & & & 0 stops & 1 stop & 2 stops & 3 stops & 4 stops & $\begin{array}{l}\text { Net } \\
\text { effect }\end{array}$ & $\begin{array}{l}\text { Total net } \\
\text { effect }\end{array}$ \\
\hline \multirow{4}{*}{$\begin{array}{l}5 \text { minute reduction } \\
\text { (on average) in } \\
\text { transit in-vehicle } \\
\text { travel time }\end{array}$} & \multirow[t]{2}{*}{ Independent } & Evening & -1.066 & -1.174 & -1.125 & -1.121 & -1.102 & -1.118 & \multirow{2}{*}{-0.432} \\
\hline & & Post home & -0.049 & 0.060 & 0.103 & 0.125 & 0.134 & 0.091 & \\
\hline & \multirow[t]{2}{*}{ Joint } & Evening & -1.323 & -0.902 & -0.707 & -0.580 & -0.446 & -0.737 & \multirow{2}{*}{-0.234} \\
\hline & & Post home & -0.074 & 0.089 & 0.156 & 0.196 & 0.222 & 0.141 & \\
\hline \multirow{4}{*}{$\begin{array}{l}5 \text { minute reduction } \\
\text { (on average) in } \\
\text { transit out-of-vehicle } \\
\text { travel time }\end{array}$} & \multirow[t]{2}{*}{ Independent } & Evening & -1.930 & -1.955 & -1.956 & -1.949 & -1.932 & -1.951 & \multirow{2}{*}{-0.824} \\
\hline & & Post home & -0.021 & 0.028 & 0.043 & 0.046 & 0.037 & 0.036 & \\
\hline & \multirow[t]{2}{*}{ Joint } & Evening & -2.410 & -1.518 & -1.139 & -0.899 & -0.655 & -1.200 & \multirow{2}{*}{-0.394} \\
\hline & & Post home & -0.103 & 0.109 & 0.225 & 0.316 & 0.408 & 0.209 & \\
\hline \multirow{4}{*}{$\begin{array}{l}50 \text { cents increase (on } \\
\text { average) in drive } \\
\text { alone cost }\end{array}$} & \multirow[t]{2}{*}{ Independent } & Evening & -4.218 & -4.165 & -4.075 & -3.980 & -3.824 & -4.067 & \multirow{2}{*}{-1.859} \\
\hline & & Post home & 0.093 & -0.114 & -0.193 & -0.245 & -0.284 & -0.175 & \\
\hline & \multirow[t]{2}{*}{ Joint } & Evening & -4.810 & -3.064 & -2.378 & -1.954 & -1.514 & -2.492 & \multirow{2}{*}{-1.033} \\
\hline & & Post home & -0.024 & 0.012 & 0.062 & 0.109 & 0.158 & 0.058 & \\
\hline
\end{tabular}

